(to densities approximately $10^{4}$ greater than solid state densities) which may become possible with the development of suitably tailored laser pulses. At these very high densities heating of the target by fusion products (both $\alpha$ particles and $14 \mathrm{MeV}$ neutrons) can occur, and significant thermonuclear gains can be expected at relatively low laser energies. If hopes raised by these first predictions are fulfilled, several useful and important differences between the scaling of reactor systems depending on magnetic and inertial confinement are anticipated.

Expenditure by the USAEC on plasma work with lasers is reported to be about $\$ 20$ million a year at present, while the very strong experimental programmes under Academicians Basov and Prokhorov at the Lebedev Institute in Moscow must involve a comparable effort.

In Britain, the Culham Laboratory of the Atomic Energy Authority first published work on the laser heating of isolated hydrogen pellets in 1967 ; plasmas produced by lasers have subsequently been used for filling magnetic traps. Important contributions to the laserplasma field have also been made by several of the universities, notably Belfast, Essex and Hull.-I. J. S.

\section{Cyg X-3 Blows Up}

RADIO astronomers have had a busy time these past two weeks. On Saturday, September 2, at 2200 UT observers at the Algonquin Radio Observatory in Ontario recorded that the X-ray source Cygnus X-3 had suddenly become one of the brightest sources in the radio sky at their observing wavelength of $2.8 \mathrm{~cm}$, an astonishing discovery considering that the first detection of radio emission from Cyg X-3 was announced only two months ago.

Since the Canadian discovery communications between observatories have been at a high pitch. By early Sunday morning the outburst from Cyg X-3 had been confirmed at seven wavelengths from $1.9 \mathrm{~cm}$ to $21 \mathrm{~cm}$ following an immediate alert of other radio observatories by the observers in Canada. In France observers at the Radio Observatory of Nancay have estimated the distance of Cyg X-3 by observing that the $21 \mathrm{~cm}$ hydrogen line emission from Cyg X-3 shows absorption due to the spiral arm at $8 \mathrm{kpc}$, but no absorption that could be due to the $11 \mathrm{kpc}$ arm (1 kpc=3,260 light years). Cyg X-3 must therefore be between $8 \mathrm{kpc}$ and $11 \mathrm{kpc}$.

It is, of course, early days yet, but it is clear that an unprecedented eruption of some kind has taken place on Cyg X-3. The more optimistic will point out that we are overdue for another supernova-the last to be recorded in our Galaxy was a good three hundred years ago. But so far there have been no reports of the optical detection of Cyg X-3--possibly not surprising because that part of the Cygnus constellation is heavily obscured.

The signal recorded at Algonquin was 22 flux units (1 flux unit $=10^{-26} \mathrm{~W} \mathrm{~m} \mathrm{~m}^{-2} \mathrm{~Hz}^{-1}$ ). Before then the strongest signal from Cyg X-3 at that wavelength had been about 0.5 flux units, and the signal had often been less than that. But it was clear from observations made earlier this year and last year at the Westerbork observatory in Holland-where the radio emission was first detected and at the National Radio Astronomy Observatory in West Virginia that the signal from Cyg $\mathrm{X}-3$ can be extremely variable on a time scale of hours and that it could therefore be classed as an unusual source.
There seems to be no evidence so far that Cyg X-3 is behaving like a pulsar.-By our Astronomy Correspondent.

\section{Dapsone and leprosy}

THE causal agent of leprosy, Mycobacterium leprae, cannot be cultured on cell-free bacterial media. The discovery that leprosy bacilli could survive and multiply in the foot-pads of normal mice, and especially in those rendered immunologically deficient by thymectomy and whole-body irradiation, has revolutionized research into the pathogenesis and treatment of the disease. Of the drugs that have been used in the treatment of leprosy, dapsone (4,4-diaminodiphenyl sulphone, DDS) is still the standard agent, and its minimal inhibitory concentration can be estimated in vivo by measuring its concentration in the serum of mice fed with the minimal effective dose required to inhibit bacterial multiplication in the footpads. It has recently been found that dapsone is converted in man to monoacetyldapsone (MADDS) by the same enzyme system responsible for acetylating isoniazid and sulphamethazine. Indeed, the acetylation of isoniazid is important in the inactivation of the drug, and those subjects who are completely deficient in the enzyme may develop polyneuritis because of high levels of circulating isoniazid. But, unlike acetylisoniazid and acetylsulphamethazine, MADDS is rapidly deacetylated in man. The half-life of dapsone seems to be unrelated to its speed of acetylation.

In the article by Ellard et al. on page 159 of this week's issue of Nature, an attempt is made to determine whether the rate of acetylation of dapsone is of prognostic importance in the treatment of leprosy. It has been found that patients of low acetylator phenotype can respond favourably to isoniazid when on suboptimal regimes, and Ellard and his colleagues have made use of this observation to test the acetylation status of two groups of patients with leprosy. One group had received a hundredth of the conventional dose of dapsone in a pilot chemical trial, and yet had responded initially as well as those on the usual dose. The other group consisted of patients who had relapsed after many years of treatment with conventional doses; the relapse was due to the emergence of strains of leprosy bacillus resistant to dapsone.

The acetylator phenotype of the patients was assessed in terms of their capacity to acetylate isoniazid. The level of isoniazid in the plasma eight hours after its oral administration and the amount of acetylisoniazid excreted in the urine during the first two hours were the measurements used. In the first group of patients there were some rapid acetylators, and these responded to treatment during the first months as satisfactorily as did the slow acetylators. In the second group there were also some rapid acetylators, but in both the slow and the rapid acetylators, bacilli were able to multiply in the food-pads of mice given an oral dose of dapsone equivalent to the conventional dose in man.

Ellard and his colleagues induce from these results that the rate of acetylation of dapsone is not a significant prognostic factor in the treatment of leprosy. It may be that MADDS has intrinsic antileprotic activity, but, as it is rapidly deacetylated to dapsone both in man and mouse, its therapeutic efficiency cannot at present be established.-M. I. 